\title{
Implementation of a Formative Objective Structured Clinical Exam to assess self evaluation in a rural BSN-DNP program
}

\author{
Jennifer Lynn Rogers; Katy Garth \\ School of Nursing, Murray State Univeristy, United States
}

Received: August 6, 2020

DOI: $10.5430 /$ jnep.v10n12p69
Accepted: September 16, 2020 Online Published: October 18, 2020

URL: https://doi.org/10.5430/jnep.v10n12p69

\begin{abstract}
Background and objective: The role of self-assessment in competency-based education has been controversial. The Objective Structured Clinical Exam (OSCE) has been used to assess competencies across the health professions. However, exploring the role of the OSCE as a method of self-assessment for nursing students has been limited. Objective: Implementation of a low cost pilot OSCE in a rural BSN-DNP program to explore graduate nursing students perceived self-evaluation of competencies to their actual OSCE performance.

Methods: Eight students enrolled in a small, rural Bachelor of Science and Nursing to Doctorate of Nursing Practice (BSNDNP) program in the Family Nurse Practitioner (FNP) specialty track were required to complete an OSCE. Graduate students participating in the OSCE completed a Self-Assessment of Competency questionnaire prior to performing the OSCE and the results were compared to their actual performance on the OSCE. Using available resources, undergraduate students in the BSN program at the institution were utilized as standardized patients.

Results: Students perceived self-assessment of competence rated higher than their actual performance in subjective and objective data collection and implementation of a plan. Students' actual performance was superior to their perceived self-assessment regarding communication with the patient.

Conclusions: Without competency-based self-assessments, students can be unaware of their strengths and weaknesses. The OSCE is an instrument that provides faculty and students with objective measures of self-evaluation and should be considered as a component of competency-based education in rural nursing institutions.
\end{abstract}

Key Words: Objective Structured Clinical Exam, Advanced practice nursing education, Competency based education, Selfassessment

\section{INTRODUCTION}

Competency is defined as actions that demonstrate mastery at work and are used to establish work standards and create strategies to depict individuals and teams. ${ }^{[1]}$ Competency encompasses learned knowledge, behaviors, communication, skills, and clinical reasoning. ${ }^{[2]}$ Demonstration of compe- tency is a mandated requirement of university accrediting agencies and state boards of nursing, illustrating its importance in nursing education. Competence is a critical factor in the transition from student to advanced practice nurse. ${ }^{[3]}$

The role of self-assessment in professional competence has not clearly been defined. In research that addressed objec-

*Correspondence: Jennifer Lynn Rogers; Email: jrogers37@murraystate.edu; Address: School of Nursing, Murray State Univeristy, United States. 
tive methods of measuring self-assessment, results have been mixed. ${ }^{[4]}$ However, the role of learned self-assessment within competency based education cannot be overlooked. ${ }^{[5,6]}$ It is recognized that students who correctly identify their own strengths and weaknesses self-direct their learning and have improved outcomes after graduation. ${ }^{[4]}$ Even so, research has found a disconnection between students' self-assessment of competence and actual clinical performance across the health professions. ${ }^{[3,4,6]}$ The role of self-evaluation in competencybased nursing education requires further exploration. ${ }^{[3,4,6]}$

As graduate Advanced Practice Registered Nurse (APRN) faculty focus on the development of competence-based curricula, assessment of competency is critical. The Objective Structured Clinical Exam (OSCE) has been used to evaluate clinical competence within the health professions. ${ }^{[5]}$ It has provided graduate nursing students and faculty an opportunity to validate their aptitude through an objective measure of clinical competence.

The OSCE was introduced in the 1970s by Dr. Ronald M. Harden in order to address the unreliability and lack of generalizability of the traditional forms of assessment in medical education. ${ }^{[7,8]}$ It was established in nursing in the early 2000s. ${ }^{[9,10]}$ The examination consists of multiple standardized stations at which students must complete specific clinical tasks, involving patient actors (standardized patients) or simulated encounters. ${ }^{[8]}$ Implementation of the OSCE can be resource intensive, however, utilization of the standardized patient in the OSCE offers clinical learning experiences in a controlled, protected environment promoting clinical judgement and reasoning. ${ }^{[11]}$ Both of these are key components of competency-based education.

While the OSCE can be used as an assessment of clinical competency for graduate nursing students, barriers for implementation exist. The financial cost and that of faculty time for development and implementation of the OSCEs can limit its use in small, rural programs. ${ }^{[11]}$ However, incorporation of the OSCE should be considered as it provides students' with effective opportunities for learned self-assessment and is a method for faculty to evaluate required competencies. ${ }^{[3,5]}$

Literature indicates that the OSCE can be used as an assessment of clinical competency in graduate nursing students. ${ }^{[12]}$ Its role in the ability to encompass learned self-assessment skills continues to be explored across health professions. ${ }^{[5]}$ The objective of this project is to demonstrate how the implementation of low cost pilot OSCE in a small rural institution can be used to evaluate graduate nursing students' perceived self- assessment of competency to their actual clinical performance on the OSCE.

\section{LITERATURE REVIEW}

Objective Structured Clinical Exam and/or OSCES was searched using CINAHL, Cochrane Central Registrar of Controlled Trials and Cochrane Database of Systematic Reviews databases. The search was further narrowed using key words advanced practice nursing education, competency based nursing education, and self-assessment for the years 2014-2019, identifying 20 articles. The review was divided into four categories: national guidelines, validity of the OSCE, barriers to OSCE implementation and the role of self-assessment.

\subsection{National guidelines}

Since 2009, The National Organization of Nurse Practitioner Faculties (NONPF) has identified core competencies for all nurse practitioners. These competencies have been recognized by institutional accrediting agencies, and delineate practice competencies required upon graduation from advanced practice programs. ${ }^{[13]}$ Within the domains of Common Core Competencies for the Advance Practice Nurse, the NONPF includes patient care, knowledge, practice based learning and, communication and professionalism. ${ }^{[13]} \mathrm{Un}$ der the domain, Practice-Based Learning and Improvement, continuous assessment of one's own strengths and weaknesses in knowledge and skills and actively seeking opportunities for continuous improvement is a required competency of the graduate nurse practitioner. ${ }^{[13]}$ NONPF specifically targets the students' ability to self-evaluate as an integral part of competency-based education for the advanced practitioner. The OSCE has been used as an effective assessment method to measure competence in the areas of skills and knowledge. ${ }^{[14,15]}$ Its role in self-assessment has been explored across the health professions with mixed correlations between student's perceived self-assessment and actual performance on the OSCE. ${ }^{[4,5,15]}$

\subsection{Validity of the OSCE}

Schools of medicine have been using the Objective Clinical Structured Examination (OSCE) as a measure for clinical competency since 1975. ${ }^{[9]}$ The OSCE is widely accepted as an assessment method for measuring clinical judgement and reasoning. ${ }^{[10]}$ Medical schools have used the OSCE to measure communication skills, knowledge, technical skills and behaviors. ${ }^{[6,7,15]}$ Most notably, the OSCE is now a component of entry-to-practice licensing examinations for the United States Medical Licensing Examination, the Canadian Pharmacist Qualifying Examination, and the Medical Council of Canada Qualifying Examination. ${ }^{[8]}$

Psychometric testing is vital to gauge the effectiveness and consistency of an assessment tool. In literature examining the reliability and validity of an OSCE, several factors of 
OSCE implementation have been examined: number of stations, training of standardized patients, global rating score and inter-rater reliability. ${ }^{[9,12,14,16-18]}$ Research methods to examine psychometrics include descriptive studies, quantitative designs, and systematic reviews. ${ }^{[17]}$ The quality of studies varied, yet in the majority of studies reviewed, validity and reliability were present when examining the OSCE as a means of assessment to evaluate competency-based education. ${ }^{[17]}$ Through the literature it is evident that the OSCE is a valid method of clinical assessment, however certain factors should be considered prior to implementation. Research suggests that validity and reliability increase with 8-12 stations, the use of trained standardized patients, and two faculty evaluators at each station. ${ }^{[14,16-18]}$ Consideration of planning regarding the development, performance, and assessments desired should be explored prior to implementation.

\subsection{Barriers to OSCE implementation}

While OSCEs have been used in schools of medicines since 1975, their emergence in nursing has been sporadic. The OSCE, consisting of 8-10 stations, simulation encounters, multiple faculty and management of standardized patients leads to a very resource intensive method of assessment. ${ }^{[3,9,10]}$ Therefore, the introduction of an OSCE within many nursing programs has been limited due to the high cost or scope of resources required for implementation. ${ }^{[19]}$

In a literature review examining the value of the OSCE in nursing programs, only 5 of 204 articles reviewed from 19822018 addressed the cost of the OSCE. ${ }^{[11]}$ The cost of the OSCEs ranged from 75 to 275 US dollars per student. ${ }^{[11]}$ Simulation labs, advanced technology and funding for standardized patients are considerations for implementation of an OSCE within educational programs. High costs, high resource use and the large number of faculty needed are certainly barriers for implementation in small, rural advanced practice programs. ${ }^{[4,19]}$

\subsection{The role of self-assessment}

Students' ability to accurately evaluate their knowledge and skills is a critical component of competence based learning as emphasized by NONPF's statement that the Advanced Practice Nurse (APN) competencies should include continual assessment of their strengths and weaknesses. Students who are able to recognize their weaknesses and act accordingly have improved patient outcomes. Over confidence related to erroneous self-assessment can lead to negative outcomes. ${ }^{[4]}$ Research is mixed in its view of the impact of self-assessment by students. A semi-experimental study conducted to evaluate the effects of simulation-based learning on self-efficacy and performance in first year nursing stu- dents' demonstrated decreased self-competence scores after the simulation was completed. ${ }^{[20]}$ This may be due to inconsistencies in self-assessment instruments. ${ }^{[4]}$ Self-assessment has been measured by a variety of instruments that range from a few categories and items measured on a 4 point Likert scale to 40 plus item inventories. ${ }^{[4]}$ Without a validated tool to measure OSCE outcomes or self-assessment, research results have demonstrated negative and positive correlations between self-assessment and OSCE performances. ${ }^{[3]}$

Research addressing the role of self-assessment in nursing has been limited. A systematic review comparing selfassessment with OSCEs using a pre self-assessment, followed by the OSCE within health professions found that of the 18 studies reviewed, only 2 were specific to nursing. Only 8 of the eighteen were conducted within the United States. ${ }^{[4]}$ Findings of the review indicated that self-assessment instruments are not comparable to performance on the OSCE. High performers often underestimate their ability and low performers are noted to overestimate their ability. ${ }^{[4,6]}$ With schools now being held more accountable for the competence of individuals upon graduation, educators are becoming aware of the importance of teaching students how to accurately assess their own performance. ${ }^{[4]}$ Further exploration of the instruments used for self-assessment is indicated.

\section{MeTHODS}

A BSN-DNP Family Nurse Practitioner tract program in the Southeastern region of the United States was chosen for the pilot OSCE. The chosen institution was accredited by the Commission on Collegiate Nursing Education (CCNE) with approximately 6-12 students in each graduating class. Family nurse practitioner students within the BSN-DNP program completed their required clinical hours outside of the institution under the guidance of an assigned preceptor. Faculty members made visits to these sites for student evaluations. There was no opportunity for observed standardized simulated patient encounters by the faculty within this institution. Implementation of the OSCE gave faculty the opportunity to observe student's role as a future practitioner in a controlled environment and assisted faculty to assess for required competencies. The project was submitted for review by the Institutional Review Board at the participating location and was determined to be exempt from review.

Prior to the start of the OSCE each participating graduate student completed a self-assessment of their own clinical competency by filling out the Pre-OSCE evaluation. The Pre-OSCE competency questionnaire was developed from National Organization of Nurse Practitioner Faculties (NONPF) required competencies for the advanced practice nurse. Evaluated competencies included subjective and objective data collec- 
tion, decision making skills and development and implementation of the plan of care. The students were then asked to self-assess each area as competent (I feel self-directed and independent in providing safe and quality care), fairly competent (in most cases I feel I am able to provide safe and quality care), moderate (I requires consistent guidance to provide safe and quality care) and considerable (I require assistance to provide safe and quality care).

After completion of the self-assessment questionnaire students began the formative OSCE assessment. Eight third year FNP students completed the OSCE as part of a formative assessment. Prior to the day of the OSCE, the FNP students were provided with instructions for the exercise, and an example of the grading rubric was sent to students via email. The grading rubric completed by 2 observing faculty assessed subjective and objective data collection, decision making skills, communication and development and implementation of a plan.

Faculty within the nursing department were asked to assist with the OSCE. Two faculty were assigned to each enacted scenario. Of the two faculty evaluators, one was currently active in clinical practice. Seniors enrolled in the undergraduate BSN program volunteered to play the role of the standardized patients. The undergraduate BSN students were sent an email requesting volunteer participation in the OSCE. There was no benefit given to those undergraduate students who volunteered for the role of the standardized patient. A signature was required by the undergraduates stating that they were aware no benefits would be given, participation was voluntary and they could withdraw from the project at any time.

The undergraduate students were brought in a week prior to the OSCE and were provided with a twenty minute training regarding the signs and symptoms of the scenarios they would be enacting the following week. During the training, volunteers were presented with the clinical scenarios. Information was given to each volunteer, identifying the age and sex of the patient they would be role playing. In addition the disease process, chief complaint, health history, present signs and symptoms, physical exam findings, vital signs were provided. To reduce repeat exams and possible trauma to the volunteers, images of physical findings were placed on laminated cards for the graduate students to view. For example, one chief complaint was an ear infection. Laminated cards demonstrating the abnormalities of the ear exam were shown to the graduate students, when they indicated that they were going to examine in the standardized patient ears.

The formative OSCE included three clinical simulations stations to be completed with the assistance of the under- graduate students as standardized patients and two faculty evaluators. The scenarios presented related to hypertension, vaginal discharge and otitis media. Graduate students were evaluated by two faculty, one of whom was a Family Nurse Practitioner using a rubric. Each BSN-DNP student had 15 minutes to complete the history and physical examinations of each scenario and then 15 minutes to write a subjective, objective, assessment and plan (SOAP) note before switching to the next station. The fourth OSCE was a written OSCE that consisted of ten clinical cases ranging from writing a prescription, interpreting radiology results or managing abnormal mammogram results. The students were given one hour to complete this. The written OSCE was proctored by a faculty member.

After all eight students had completed all four stations, the OSCEs were discussed in a debriefing session immediately following with the group. The students were provided with the faculty graded rubrics for each completed scenario. The undergraduate students were invited to stay and participate in the debriefing session, discussing their concerns and providing feedback to the examining advanced practice students. The pre-OSCE self-assessment questionnaire was compared to the graduate students' performance on the OSCE. Upon completion of the debriefing, both undergraduate and graduate students completed an anonymous post OSCE evaluation.

\section{RESUlts}

As shown in Table 1, five of the eight students evaluated themselves as fairly competent (in most cases I feel I am able to provide safe and quality care) to competent (I feel self-directed and independent in providing safe and quality care) in the areas of assessment, decision making skills/development and implementation of a plan of care. Their actual performance on the OSCE demonstrated moderate (Requires consistent guidance to provide safe and quality care) and considerable (Requires assistance to provide safe and quality care) in collection of subjective and objective data. In areas of decision making/development and implementation of plan eight of the students ranked themselves as moderate to competent but their performance on the OSCE was equal to or lower than their self-evaluation in these areas. In summary, these students perceived competence was higher than their actual performance on the OSCE.

Three of the students rated themselves as moderate in areas of subjective and objective data collection, decision making/development and implementation of plan. All three were evaluated the same or higher in collection of subjective and objective data collection by faculty on their actual clinical performance. Six of eight students rated themselves as competent in communicating with the patient, whereas two felt 
they were fairly competent in communication with patients. competence was less than their actual performance on the All eight students received high evaluations on the OSCE OSCE. in areas of communication. In summary, their perceived

Table 1. Results of Evaluated Domains: When A is greater than B, the students perceived self-assessment of competency is higher than the actual performance and vice versa when B is greater than A

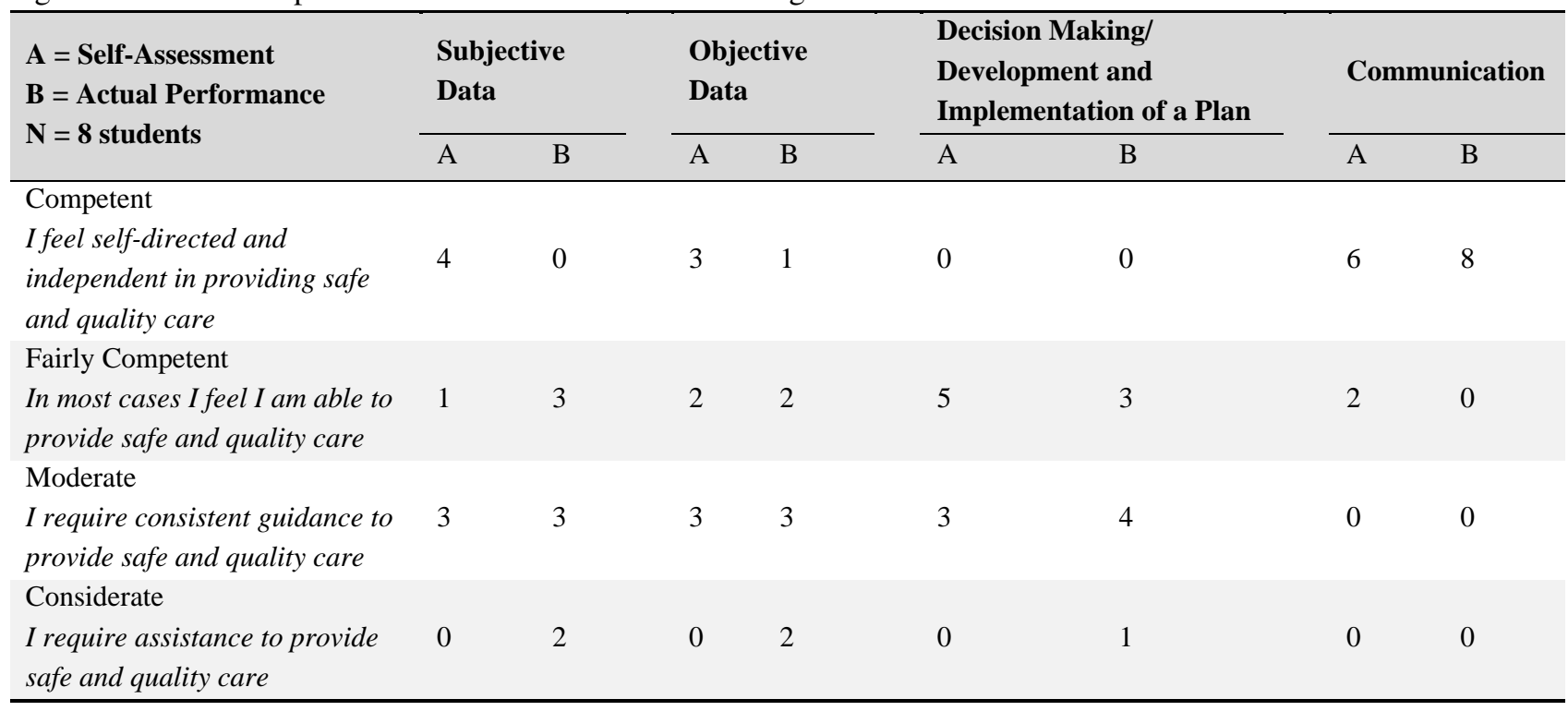

In the open-ended post OSCE evaluation, graduate students were asked how the completion of an OSCE may or may not help them in future practice as an FNP. Students stated, "it helped me to practice my critical thinking skills", "I feel the OSCE was very helpful", "and it provided feedback that was very valuable". Further comments included "it was a great tool for self-reflection on how I can improve and what areas I feel confident in" and "I realized where I stood and how I can improve". Interestingly, one student stated "it made me feel like I can actually do this. It gave me the chance to practice independently".

Undergraduate students were also asked to evaluate their experience of playing the roles of the standardized patients. In response to the question of how the OSCE may or may not help them in future practice as a student or RN, stated "I think it was an awesome experience to gauge clinical preparedness and building confidence". "I would love to have experiences like this for the undergraduate program" and "as a student I feel like I learned to be in the patient's shoes, and it helped me learn what to ask." Interestingly these students also felt that the experience augmented their education. "For hypertension patients, it increased my level of confidence because I was able to pull from the knowledge I already knew", after the experience "I feel better able to deal with hypertension patients". Finally, it improved the BSN students' awareness of the FNP role, "I was able to see a clinical experience of

Published by Sciedu Press a DNP student" and "get a better understanding of the FNP role."

\section{Discussion}

The implementation of a pilot OSCE demonstrated that a student's self-assessment may inaccurately reflect how they actually perform clinically. Whereas, several students evaluated their clinical ability as higher than they demonstrated, others saw themselves as less competent than their actual performance indicated. This difference demonstrates the strong need for objective, competency-based clinical education, which includes knowledge regarding accurate selfassessment.

Overall, the implementation of the pilot OSCEs for FNP clinical evaluation proved to be beneficial for both the graduate and undergraduate participants. Evaluation of graduate students' ability to collect a history, perform a physical assessment, communicate, implement a plan and document in a safe, controlled environment gave an opportunity for them to identify their strengths and weaknesses. For many of the students their perceived strengths and/or weaknesses were quite different than those that were identified after the OSCE. Without this knowledge students would not be able to recognize their own strengths and weaknesses.

There has been limited research completed on reducing expenditures of OSCE implementation. Richardson, Resick, 
Leonardo and Pearsall (2009) completed a study examining the role of undergraduate students playing the role of standardized patients in an OSCE. ${ }^{[19]}$ The study identified enhanced collaboration between undergraduate and graduate faculty, minimalizing costs of the OSCE, and promoted innovative learning opportunities for both graduate and undergraduate students as positive outcomes of the experience. ${ }^{[19]}$ With limited articles addressing implementation in small, rural institutions, more research is needed.

This project supports the use of OSCEs as a form of competency evaluation in a rural BSN-DNP, FNP specialty track program to assist with self-evaluation skills. While OSCEs can be costly to implement in smaller programs, they are beneficial and can be completed with minimal impact to the budget by using available resources. Using undergraduate students as the standardized patients presented opportunities for their educational growth and enhanced their educational experience.

With the knowledge that student's own self-assessment was observed as different from their actual performance, more emphasis should be placed on student self-evaluations. The use of video assisted OSCEs may contribute further to selfassessment skills. Videotaping the students' performance and allowing students to evaluate their own performance in the OSCE would allow for further self-recognition of an individual's strengths and weakness.

\section{Conclusion}

With the knowledge that student's own self-assessment was observed as different from their actual performance, more emphasis should be placed on student self-evaluation. Implementation of an OSCE is a reliable and valid method of competency-based clinical education and through careful planning and development can assist with educating students on performing accurate self-assessments. While implementation of OSCEs is often deferred due to budget concerns, it is feasible to introduce an OSCE into a rural BSN-DNP program, using available resources. Further OSCE development including the enhancement of scenarios, strategies and procedures relating to self-assessment and clinical competencies would be beneficial. The use of video assisted OSCEs may contribute further to self-assessment skills. As videotaping the students' performance and allowing students to evaluate their own performance in the OSCE would allow for further self-recognition of an individual's strengths and weakness. The success of this pilot study supports the need for further exploration of the role of OSCEs and student self-assessment.

\section{Limitations}

Although the students were evaluated by two faculty, inter rater reliability was identified as a problem. Without clear instructions for faculty regarding instructions of the grading rubric, results varied considerably amongst evaluators. For example, one faculty member evaluated a student as competent in the area of assessment whereas the other faculty evaluated the student as moderate in their ability to assess the standardized patient. Inter rater reliability may have been further increased due to one faculty practicing as an FNP. As a result the evaluators may have been inherently looking at the skills and expectations differently leading to different evaluations.

Research indicates that to increase validity and reliability of an OSCE, 8-12 stations are recommended. ${ }^{[14,16-18]}$ Due to a limited number of faculty in a small, rural program, this was not feasible. It is to be noted that only having four stations may decrease the validity of the results.

For future implementation, recommendations would include introduction of a revised evaluation rubric with faculty training regarding expectations of the grading rubric to increase consistency among the evaluators therefore increasing inter rater reliability. Consideration of the ability to increase the number of stations present for the OSCE. Videotaping the students performing the scenarios would also allow the evaluators to complete the rubrics later and allow for discussion amongst among faculty regarding grading, increasing consistency of evaluations as well as provide the opportunity for student self-assessments.

\section{ACKNOWLEDGEMENTS}

The authors would like to acknowledge Dr. Belinda Fleming and her associates at the Loewenberg College of Nursing at the University of Memphis for sharing their OSCE development and process, contributing to the methodology of the pilot OSCE implementation.

\section{CONFlicts OF INTEREST Disclosure}

The author declares that there is no conflict of interest.

\section{REFERENCES}

[1] Wu XV, Enskär K, Lee CCS, et al. A systematic review of clinical assessment for undergraduate nursing students. Nurse Education Today. 2015; 35(2): 347-359. PMid:25497138 https ://doi .org/10
$.1016 / j$. nedt .2014 .11 .016

[2] Epstein RM, Hundert EM. Defining and assessing professional competence. JAMA. 2002 Jan 9; 287(2): 226-35. PMid:11779266 https://doi.org/10.1001/jama.287.2.226 
[3] Taylor I, Bing-Jonsson P, Wangensteen S, et al. The self-assessment of clinical competence and the need for further training: A crosssectional survey of advanced practice nursing students. J Clin Nurs. 2020 Feb; 29(3-4): 545-555. PMid:31714619 https ://doi.org/ $10.1111 /$ jocn. 15095

[4] Sears K, Godfrey C, Luctkar-Flude M, et al. Measuring Competence in Healthcare Learners and Healthcare Professionals by Comparing Self-Assessment with Objective Structured Clinical Examinations: A Systematic Review. JBI Database of Systematic Reviews \& Implementation Reports. 2014 Dec; 12(11): 221-72. https://doi.org/10.11124/jbisrir-2014-1605

[5] Doloresco F, Maerten-Rivera J, Zhao Y, et al. Pharmacy Students' Standardized Self-Assessment of Interprofessional Skills during an Objective Structured Clinical Examination. Am J Pharm Educ. 2019 Dec; 83(10): 7439. PMid:32001878 https://doi.org/10.5688/ ajpe 7439

[6] Graves L, Lalla L, Young M. Evaluation of perceived and actual competency in a family medicine objective structured clinical examination. Can Fam Physician. 2017 Apr; 63(4): e238-e243.

[7] Gormley G. Summative OSCEs in undergraduate medical education. Ulster Med J. 2011 Sep; 80(3): 127-32.

[8] Muthamilselvi G, Vadivukkarasi RP. Objective structured clinical examination - Emerging trend in nursing profession. International $\mathrm{J}$ of Nurs Ed. 2014 Jan-Jun; 6(1): 43-47. https://doi.org/10.5 958/j.0974-9357.6.1.009

[9] Rushforth HE. Objective structured clinical examination (OSCE): review of literature and implications for nursing education. Nurse Educ Today. 2007 Jul; 27(5): 481-90. PMid:17070622 https: //doi.org/10.1016/j.nedt.2006.08.009

[10] Traynor M, Galanouli D. Have OSCEs come of age in nursing education? Br J Nurs. 2015 Apr 9-22; 24(7): 388-91. PMid:25849236 https://doi.org/10.12968/bjon.2015.24.7.388

[11] Goh HS, Zhang H, Lee CN, et al. Value of Nursing Objective Structured Clinical Examinations: A Scoping Review. Nurse Educ. 2019 Sep/Oct; 44(5): E1-E6. PMid:30371544 https ://doi .org/10.1 097/NNE. 0000000000000620

[12] Aronowitz T, Aronowitz S, Mardin-Small J, et al. Using Objective Structured Clinical Examination (OSCE) as Education in Advanced
Practice Registered Nursing Education. J Prof Nurs. 2017 Mar-Apr; 33(2): 119-125. PMid:28363386 https://doi.org/10.1016/j. profnurs.2016.06.003

[13] Nurse Practitioner Core Competencies Content. The national organization of nurse practitioner faculties (NONPF). 2017; Available from: https ://cdn. ymaws . com/www. nonpf .org/resource/resmg r/competencies/2017_NPCoreComps_with_Curric.pdf

[14] Hongli S, Mun L, Tang D, et al. Testing the psychometric properties of objective strucured clinical examination (OSCE) in nursing education in Singapore. Singapore Nursing J. 2016 Jan-Apr; 43(1): 12-18.

[15] Ginsburg LR, Tregunno D, Norton PG, et al. Development and testing of an objective structured clinical exam (OSCE) to assess sociocultural dimensions of patient safety competency. BMJ Qual Saf. 2015 Mar; 24(3): 188-94. PMid:25398630 https : //doi .org/10 $.1136 /$ bmjqs-2014-003277

[16] Shen L, Zeng H, Jin X, et al. An Innovative Evaluation in Fundamental Nursing Curriculum for Novice Nursing Students: An Observational Research. J Prof Nurs. 2018 Sep-Oct; 34(5): 412-416. PMid:30243698 https://doi.org/10.1016/j.profnurs. 201 8.05 .002

[17] Brannick MT, Erol-Korkmaz HT, Prewett M. A systematic review of the reliability of objective structured clinical examination scores. Med Educ. 2011 Dec; 45(12): 1181-9. PMid:21988659 https://doi.org/10.1111/j.1365-2923.2011.04075.x

[18] Navas-Ferrer C, Urcola-Pardo F, Subirón-Valera AB, et al. Validity and reliability of objective structured clinical evaluation in nursing. Clinical Simulation in Nurs. 2017; 13(11): 531-543. https: //doi.org/10.1016/j.ecns.2017.07.003

[19] Richardson L, Resick L, Leonardo M, et al. Undergraduate students as standardized patients to assess advanced practice nursing student competencies. Nurse Educ. 2009 Jan-Feb; 34(1): 12-6. PMid:19104339 https://doi.org/10.1097/01 . NNE.0000343 397.26695 .89

[20] Karabacak U, Unver V, Ugur E, et al. Examining the effect of simulation based learning on self-efficacy and performance of firstyear nursing students. Nurse Educ Pract. 2019 Mar; 36: 139-143. PMid:30978577 https://doi.org/10.1016/j.nepr.2019.03 .012 